\title{
Underlying Mechanisms of Anastomotic Leakage and Systemic Recurrences in Colorectal Cancer
}

\author{
Steven Oosterling $\cdot$ Hein Stockmann • \\ Gerben van der Bij • Eric Belt • Marjolein van Egmond
}

Received: 23 January 2011 / Accepted: 20 June 2011 / Published online: 14 July 2011

(C) 2011 The Society for Surgery of the Alimentary Tract

\section{Dear Sir,}

With interest we read the recent article of Katoh et al. in which the contribution of anastomotic leakage to the risk for systemic recurrence in a series of 207 stage II colorectal cancer patients was investigated. ${ }^{1}$ In their multivariable analysis, anastomotic leakage proved the most robust independent prognostic factor for a 5-year disease-free survival. The authors address in their discussion a main factor that might contribute to increased risk of recurrences. The use of perioperative blood transfusions was reported as a risk factor for anastomotic leakage and has earlier been established as a prognostic factor for cancer recurrence. As stated by the authors, blood transfusions are associated with impaired cell-mediated immune responses which might facilitate development of (micro) metastases.

Here, we would like to discuss several other biological mechanisms that might be responsible for increased recurrences seen following anastomotic leakages. In the case of anastomotic leakage or otherwise failure of the barrier function, the gut provides a major source of endotoxins that ubiquitously circulate while leading to sepsis. Endotoxins or lipopolysaccharides (LPS), which are cell-wall constituents of Gram-negative bacteria, have been shown to enhance tumor cell adhesion and metastasis outgrowth in experimental models. ${ }^{2,3}$ Mice that received endotoxin injections showed increased metastatic burden compared to those receiving a saline injection. This increase was reflected in higher tumor cell proliferation and decreased apoptosis within lung metastases. ${ }^{2}$ We earlier

S. Oosterling $(\bowtie) \cdot H$. Stockmann $\cdot$ G. van der Bij $\cdot$ E. Belt

M. van Egmond

Kennemer Gasthuis,

Haarlem, The Netherlands

e-mail: sj.oosterling@gmail.com showed in several experimental models that the perioperative milieu itself facilitates both local and systemic tumor metastasis formation. ${ }^{4,5}$ Electron microscopic investigation demonstrated that abdominal surgery resulted in endothelial stress, leading to impaired liver vessel integrity and subsequent exposure of underlying extracellular matrix, which served as preferred adhesion sites for tumor cells leading to enhanced metastasis outgrowth. ${ }^{5}$ In accordance, in a recent in vitro study, LPS enhanced tumor cell adhesion and invasion, both fundamental processes in tumor progression. ${ }^{3}$ Moreover, continued and repetitive exposure to endotoxins, such as during abdominal sepsis, results in reduced immune responsiveness or even immune paralysis. This is exemplified by diminished capability of innate immune cells, such as macrophages, to produce the cytotoxic tumor necrosis factor $\alpha$. Since liver macrophages, the so-called Kupffer cells, have been shown to be pivotal in arresting and eliminating circulating tumor cells in liver sinusoids, ${ }^{6}$ this immunosuppression may impede an effective antitumor response. Thus, endotoxins such as LPS seem to play an important role in the observations of Katoh et al. and might be connected as one of the main responsible mechanisms for increased recurrence following anastomotic leakage. As such, the link between endotoxins and tumor recurrence is a further subject of our current investigation.

\section{References}

1. Katoh H, Yamashita K, Wang G et al. Anastomotic Leakage Contributes to the Risk for Systemic Recurrence in Stage II Colorectal Cancer. J Gastrointest Surg 2011 Jan; 15(1):120-9.

2. Pidgeon GP, Harmey JH, Kay E et al. The role of endotoxin/ lipopolysaccharide in surgically induced tumour growth in a murine model of metastatic disease. Br J Cancer 1999; 81:1311-1317. 
3. Killeen SD, Wang JH, Andrews EJ et al. Bacterial endotoxin enhances colorectal cancer cell adhesion and invasion through TLR-4 and NF-kappaB-dependent activation of the urokinase plasminogen activator system. Br J Cancer 2009; 100:1589-1602.

4. Oosterling SJ, van der Bij GJ, Bogels $\mathrm{M}$ et al. Anti-betal integrin antibody reduces surgery-induced adhesion of colon carcinoma cells to traumatized peritoneal surfaces. Ann Surg 2008; 247:85-94.
5. van der Bij GJ, Oosterling SJ, Bogels $M$ et al. Blocking alpha2 integrins on rat CC531s colon carcinoma cells prevents operationinduced augmentation of liver metastases outgrowth. Hepatology 2008; 47:532-543.

6. Heuff G, Oldenburg HS, Boutkan H et al. Enhanced tumour growth in the rat liver after selective elimination of Kupffer cells. Cancer Immunol Immunother 1993; 37:125-130. 